\title{
Decreased expression of PIAS1 and PIAS3 in essential thrombocythemia patients
}

\author{
H.-H. Hsiao ${ }^{1,2}$, Y.-C. Liu' ${ }^{1,2}$, M.-Y. Yang ${ }^{3}$, Y.-F. Tsai ${ }^{2}$, T.-C. Liu' ${ }^{1,2}$, \\ C.-S. Chang ${ }^{1,2}$ and S.-F. Lin ${ }^{1,2}$ \\ ${ }^{1}$ Faculty of Medicine, College of Medicine, Kaohsiung Medical University, \\ Kaohsiung, Taiwan \\ ${ }^{2}$ Division of Hematology-Oncology, Department of Internal Medicine, \\ Kaohsiung Medical University Hospital, Kaohsiung, Taiwan \\ ${ }^{3}$ Graduate Institute of Clinical Medical Sciences, College of Medicine, \\ Chang Gung University, Tao-Yuan, Taiwan \\ Corresponding author: S-F Lin \\ E-mail: shlin@cc.kmu.edu.tw
}

Genet. Mol. Res. 12 (4): 5617-5622 (2013)

Received November 12, 2012

Accepted September 3, 2013

Published November 18, 2013

DOI http://dx.doi.org/10.4238/2013.November.18.10

\begin{abstract}
Gain of function mutation of Janus kinase 2 (JAK2V617F) has been identified in Philadelphia-negative myeloproliferative diseases; about half of essential thrombocythemia (ET) patients harbor this mutation. The activated JAK-STAT pathway promotes cell proliferation, differentiation and anti-apoptosis. We studied the role of negative regulators of the JAK-STAT pathway, PIAS, and SOCS in ET patients. Twenty ET patients and 20 healthy individuals were enrolled in the study. Thirteen of the ET patients harbored the JAK2V617F mutation based on mutation analysis. Quantitative-PCR was applied to assay the expression of SOCS1, SOCS3, PIAS1, PIAS3. The expression levels of PIAS1 and PIAS3 were significantly lower in ET groups than that in normal individuals. There was no significant difference between $J A K 2 V 617 F(+)$ and $J A K 2 V 617 F(-)$ patients. SOCS1 and SOCS3 expression did not differ between ET patients and normal individuals, or between $J A K 2 V 617 F(+)$ and $J A K 2 V 617 F(-)$ patients. We suggest that failed negative regulators of the JAK-STAT pathway take part in the pathomechanism of ET.
\end{abstract}

Key words: Essential thrombocythemia; PIAS; SOCS 


\section{INTRODUCTION}

Essential thrombocythemia (ET) characterized by an increase in platelet count in peripheral smears and an increase in megakaryocytes in bone marrow is a clonal hematopoietic disease and one of the myeloproliferative neoplasms (MPN) (Campbell and Green, 2006; Tefferi, 2006). Although ET has characteristic clinical manifestations, the real pathomechanism of the disease is still unclear. In 2005, a novel somatic mutation of Janus kinase 2 (JAK2) was identified among Philadephia chromosome-negative MPNs, including the majority of polycythemia vera (PV) and approximately half of ET and primary myelofibrosis (MF) (Baxter et al., 2005; Jones et al., 2005; Kralovics et al., 2005). The gain-of-function mutation of JAK2 in codon 617 (JAK2V617F) activates the JAK-STAT pathway, causing cell proliferation, differentiation and anti-apoptosis effects and eliciting the MPN phenotype in animal studies (Levine et al., 2005; Wernig et al., 2006).

In addition, dysfunctional signaling in pathways beyond the JAK-STAT pathway has also been noted in these diseases (Oh and Gotlib, 2010). Abnormal expression of negative regulators of JAK-STAT, such as dysregulation of suppressors of the cytokine signaling (SOCS) family and STAT3 have also been found in some studies (Hookham et al., 2007; Teofili et al., 2008; Senyuk et al., 2009). These results suggest an abnormal function of JAK-STAT signal transduction other than the JAK2V617F mutation.

In the current study, we analyzed the negative regulators of the JAK-STAT pathway, including the SOCS family and protein inhibitors of activated STAT (PIAS) family in our ET patients to see whether there is any difference between patients and normal individuals. We also compared patients with and without the $J A K 2 V 617 F$ mutation to get more information about the pathomechanism of the disease.

\section{MATERIAL AND METHODS}

\section{Patients}

A total of 20 ET patients, who were treated in our ward, along with 20 healthy individuals for comparison, were enrolled in the study. The study was approved by the hospital ethics committee. The Polycythemia Vera Study Group criteria were used for the diagnosis of ET (Murphy, 1999).

\section{Cells}

Granulocytes were isolated from peripheral blood after density gradient centrifugation. DNA was extracted from the granulocytes using a DNA kit. Total RNA was extracted from the leukocytes by TRIzol reagent method (Invitrogen, Carlsbad, CA, USA). The cDNA was generated from the input of 2 mg RNA with Moloney murine leukemia virus reverse transcriptase (Pormega, Madison, WI, USA).

\section{Survey of JAK2V617F and MPLW515L/K mutations}

The JAK2V617F mutation was identified by the amplification refractory mutation system (ARMS) (Jones et al., 2005). Briefly, DNA samples were genotyped by a tetra-primer of the ARSA assay, which used two primer pairs to amplify the normal and mutant sequences, plus a positive control band in a single polymerase chain reaction (PCR). The products were 
resolved on $2.5 \%$ agarose gels and visualized by ethidium bromide staining. MPLW515L/K was also analyzed in ET patients with the PCR primer set 5'-GAAGTCTGACCCTTTTTGTCTC-3' forward and 5'-AGAGTGTAAGGAGCCGCGG-3' reverse. The PCR products were analyzed by sequencer to detect the MPLW515L/K mutation.

\section{Quantitative-PCR for expression of PIAS1, PIAS3, SOCS1 and SOCS3}

The expression of PIAS1, PIAS3, SOCS1 and SOCS3 was evaluated by quantitative real-time PCR (Q-PCR). Each sample was examined at steady state and assayed in duplicate with the ABI 7700 Sequence Detector (Applied Biosystems, Foster City, CA, USA). The average of the duplicate assays was obtained and normalized to the average amount of beta-actin for each sample. The amount of PIAS and SOCS genes was normalized to the endogenous reference beta-actin gene to obtain the relative threshold cycle $(\Delta \mathrm{Ct})$, and gene expression level was calculated by the difference in the $\Delta \mathrm{Ct}$ (i.e., $2^{-\Delta \mathrm{Ct}}$ ).

The specific primer sets and TaqMan probe were designed using Primer Express software 1.5 (Applied Biosystems) and synthesized by Applied Biosystems. The primer set for SOCS1 was as follows: 5'-TCCTCCGCGACTACCTGA-3' forward and 5'-ATGGTTCC AGGCAAGTAATAACA-3' reverse with the probe: 6FAM-CCTTCCAGATTTGACCGGCA GC. The primer set for SOCS3 was as follows: 5'-GTCAACGGCCACCTGGA-3' forward and 5'-CCTTTACCCCTTAAAGCGG-3' reverse with the probe: 6FAM-GAGTTCCTGGA CCAGTACGAT.Theprimersetfor $\beta$-actin was as follows:5'-GGCCAACCGCGAGAAGAT-3' forward and 5'-CGTCACCGGAGTCCATGAG-3' reverse with the probe 6FAM-AGCCAT GTACGTTGCTAT. Commercial primers and probes were used for PIAS1 (Assay ID: Hs00184008_m1) and PIAS3 (Assay ID: Hs00180666_m1) from Applied Biosystems.

\section{Statistics}

Statistic analysis was performed by SPSS version 14 for Windows (SPSS Inc., Chicago, IL, USA). The Student $t$-test was used for continuous variables between 2 groups and ANOVA was used to compare continuous variables between 3 groups with post examination if the $\mathrm{P}$ value was significant. $\mathrm{P}<0.05$ was considered to be significant.

\section{RESULTS}

Among the 20 patients, 9 were males and 11 were females, with a mean age of 50.6 years. The survey of $J A K 2 V 617 F$ and $M P L W 515 L / K$ mutations in ET patients showed 13 of them $(65 \%, 13 / 20)$ harbored the $J A K 2 V 617 F$ mutation, while 7 of them $(35 \%, 7 / 20)$ had no mutations. Neither group had the MPLW515L/K mutation.

For PIAS1 and PIAS3 expression, Q-PCR showed significantly lower expression levels in ET patients compared to normal individuals $(\mathrm{P}=0.0027$ and $\mathrm{P}=0.0102$. respectively; Figure 1). However, there was no difference in PIAS1 and PIAS3 expression between $J A K 2 V 617 F(+)$ and JAK2V617F (-) ET patients (Figure 1). JAKV617F $(+)$ ET also had significantly lower levels of PIAS1 and PIAS3 compared to normal individuals, while $J A K 2 V 617 F$ (-) ET showed no significant difference compared to normal individuals. 
Considering the SOCS1 and SOCS3 expression levels, there was neither a difference between ET patients and individuals, nor a difference between $J A K 2 V 617 F(+), J A K 2 V 617 F$ (-) and normal individuals (Figure 2).
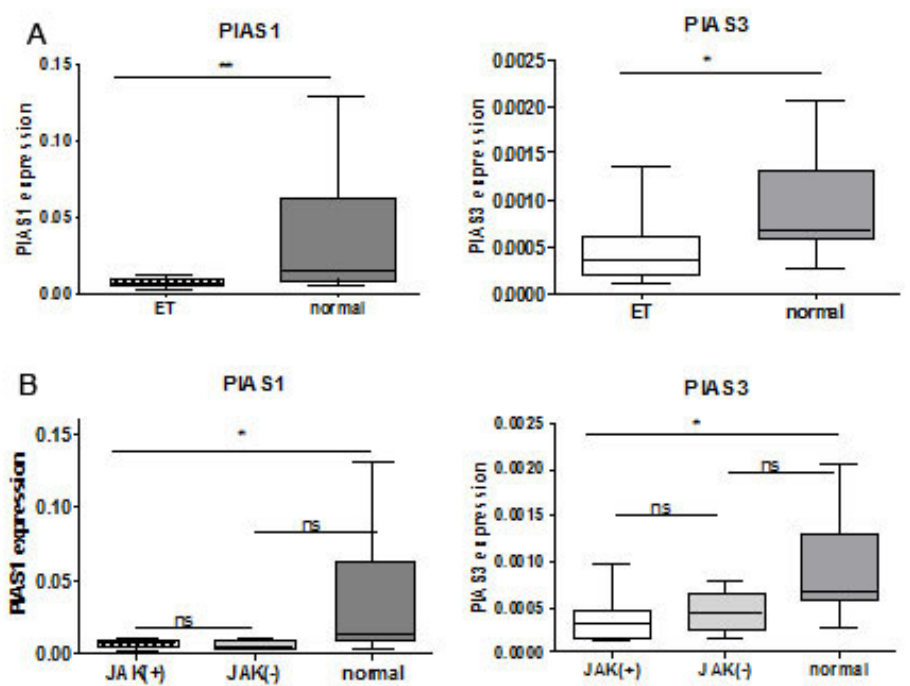

Figure 1. Expressions of PIAS1 and PIAS3. A. Comparison between ET patients and normal individuals. B. Comparison between JAK2 V617F-positive, -negative patients and normal individuals. The mean and standard deviation were represented in the figures. The level, box and bar indicate the mean, standard deviation and range of each group, respectively. ${ }^{*} \mathrm{P}<0.05 ; * * \mathrm{P}<0.01 ; \mathrm{ns}=$ no significant; JAK $=J A K 2$ V617F.
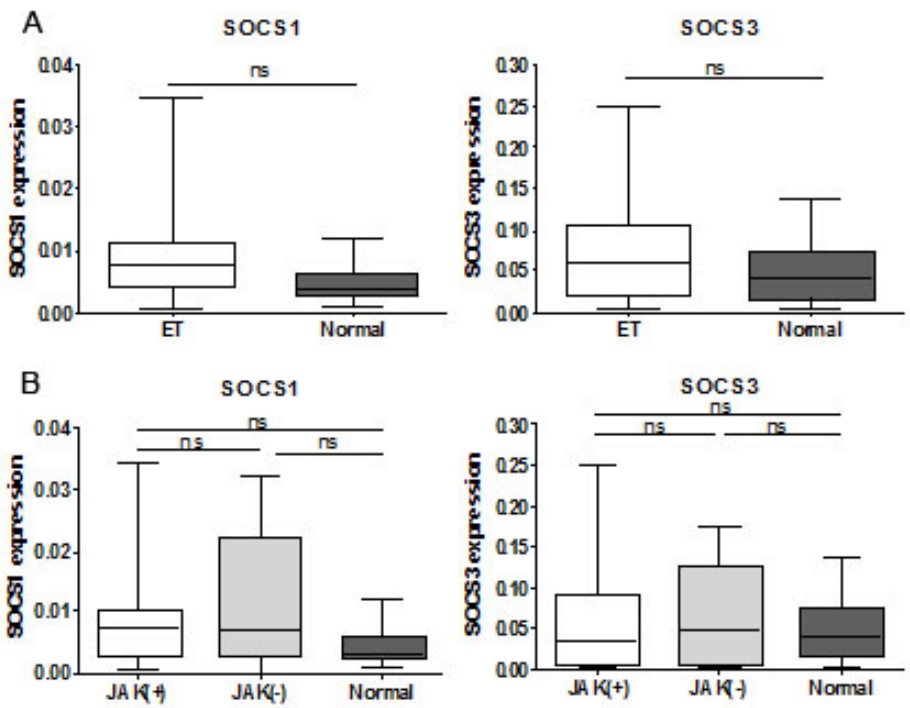

Figure 2. Expressions of SOCS1 and SOCS3. A. Comparison between ET patients and normal individuals. B. Comparison between JAK2 V617F-positive, (-) negative patients and normal individuals. The mean and standard deviation were represented in the figures. The level, box and bar indicate the mean, standard deviation and range of each group, respectively. $\mathrm{ns}=$ no significant; JAK $=J A K 2 \mathrm{~V} 617 \mathrm{~F}$. 


\section{DISCUSSION}

The $J A K 2 V 617 F$ mutation has become important for diagnosis and also clinical manifestation in Ph-negative MPNs, since it was discovered (Campbell and Green, 2006; Tefferi, 2006; Kilpivaara and Levine, 2008; Beer, 2011). It has been shown that a majority of Phnegative MPNs harbor this mutation. Our study was similar to previous reports that showed around 50-60\% of ET patients with the mutation (Hsiao et al., 2007; Ohyashiki et al., 2007). Compared to JAK2V617F, another rare mutation $(<5 \%)$, the $M P L W 515 L / K$, was also noted in ET (Ma et al., 2011). Our results showed no such mutation in the patients studied, which would not impact the results (Patnaik et al., 2010).

JAK-STAT pathway activation by tyrosine phosphorylation plays critical roles in cytokine signaling in hematopoietic cells (Valentino and Pierre, 2006). Several tyrosine phosphatases are implicated in the negative regulation of JAK-STAT signaling. The PIAS family, consisting of PIAS1, PIAS3, PIASx and PIASy in mammals, can interact with STAT, which results in inhibiting STAT-mediated gene activation (Shuai, 2006). A decrease in PIAS expression has been noted in hematologic diseases (Ueda et al., 2003; Zhang et al., 2002). We showed here that the expression of PIAS1 and PIAS3 was significantly lower in ET patients. The fact that $J A K 2 V 617 F(+)$ had a significantly lower level than did normal individuals, while $J A K 2 V 617 F(-)$ showed no difference, could imply a different mechanism between them. However, more studies with a larger scale of patients are warranted for a clear conclusion. PIAS1 and PIAS3 interact with STAT1 and STAT3, respectively, and inhibit STATs activation (Valentino and Pierre, 2006; Shuai, 2006). Though the JAK2/STAT5 pathway is supposed to be the major mediator in MPNs, some studies suggest that other mediators also take part in the disease. Teofili et al. (2007), in the study of bone marrow biopsy samples, showed that ET patients had increased pSTAT3 and reduced pSTAT5 expression, while PV had both increased pSTAT3 and pSTAT5 expression. In a murine model, Senyuk et al. (2009) also demonstrated an upregulation of STAT3 in ET regardless of JAK2V617F status. These results implied that STAT3 may be an important signal in ET patients. Lower levels of PIAS mRNA in our study supported a failed negative regulation of STAT3 independent of JAK2V617F mutation. It implied that PIAS negative regulators may participate in the disease.

SOCS proteins are a family of at least eight members that have negative regulation effects on JAK-STAT pathway (Valentino and Pierre, 2006; Croker et al., 2008). They suppress signaling either by inhibiting the JAK activity or by competition with STAT on the receptors. They could be stimulated by cytokines. In our study, the negative regulation of SOCS1 and SOCS3 seemed to have failed to inhibit the JAK-STAT pathway as there was no difference between ET patients and normal individuals. Some studies have also found dysfunction of SOCS proteins by hypermethylation in MPNs (Fourouclas et al., 2008; Teofili et al., 2008). Moreover, the negative mechanism of SOCS3 could become a positive one to promote cell proliferation in the presence of $J A K 2 V 617 F$ mutation (Hookham et al., 2007). The discrepancy between our data and previous reports about SOCS expression may come from the selection of patients and the small number of patients in the study.

Though our patient sample was small, this is the first report of dysregulated, lower expression of PIAS1 and PIAS3 in ET patients and of PIAS3 expression being unrelated to $J A K 2 V 617 F$ mutation. These data imply that the failed negative regulators of the JAK-STAT pathway may take part in the pathomechanism of essential thrombocythemia. 


\title{
ACKNOWLEDGMENTS
}

\author{
Research supported by Kaohsiung Medical University, Taiwan (grant \#M098022).
}

\section{REFERENCES}

Baxter EJ, Scott LM, Campbell PJ, East C, et al. (2005). Acquired mutation of the tyrosine kinase JAK2 in human myeloproliferative disorders. Lancet 365: 1054-1061.

Beer PA (2011). The pathogenesis of essential thrombocythemia. Curr. Opin. Hematol. 18: 323-329.

Campbell PJ and Green AR (2006). The myeloproliferative disorders. N. Engl. J. Med. 335: 2452-2466.

Croker BA, Kiu H and Nicholson SE (2008). SOCS regulation of the JAK/STAT signalling pathway. Semin. Cell Dev. Biol. 19: 414-422.

Fourouclas N, Li J, Gilby DC, Campbell PJ, et al. (2008). Methylation of the suppressor of cytokine signaling 3 gene (SOCS3) in myeloproliferative disorders. Haematologica 93: 1635-1644.

Hookham MB, Elliott J, Suessmuth Y, Staerk J, et al. (2007). The myeloproliferative disorder-associated JAK2 V617F mutant escapes negative regulation by suppressor of cytokine signaling 3. Blood 109: 4924-4929.

Hsiao HH, Yang MY, Liu YC, Lee CP, et al. (2007). The association of JAK2V617F mutation and leukocytosis with thrombotic events in essential thrombocythemia. Exp. Hematol. 35: 1704-1707.

Jones AV, Kreil S, Zoi K, Waghorn K, et al. (2005). Widespread occurrence of the JAK2 V617F mutation in chronic myeloproliferative disorders. Blood 106: 2162-2168.

Kilpivaara O and Levine RL (2008). JAK2 and MPL mutations in myeloproliferative neoplasms: discovery and science. Leukemia 22: 1813-1817.

Kralovics R, Passamonti F, Buser AS, Teo SS, et al. (2005). A gain-of-function mutation of JAK2 in myeloproliferative disorders. N. Engl. J. Med. 352: 1779-1790.

Levine RL, Wadleigh M, Cools J, Ebert BL, et al. (2005). Activating mutation in the tyrosine kinase JAK2 in polycythemia vera, essential thrombocythemia, and myeloid metaplasia with myelofibrosis. Cancer Cell 7: 387-397.

Ma W, Zhang X, Wang X, Zhang Z, et al. (2011). MPL mutation profile in JAK2 mutation-negative patients with myeloproliferative disorders. Diagn. Mol. Pathol. 20: 34-39.

Murphy S (1999). Diagnostic criteria and prognosis in polycythemia vera and essential thrombocythemia. Semin. Hematol. 36: 9-13.

Oh ST and Gotlib J (2010). JAK2 V617F and beyond: role of genetics and aberrant signaling in the pathogenesis of myeloproliferative neoplasms. Expert Rev. Hematol. 3: 323-337.

Ohyashiki K, Aota Y, Akahane D, Gotoh A, et al. (2007). JAK2(V617F) mutational status as determined by semiquantitative sequence-specific primer-single molecule fluorescence detection assay is linked to clinical features in chronic myeloproliferative disorders. Leukemia 21: 1097-1099.

Patnaik MM, Lasho TL, Finke CM, Gangat N, et al. (2010). MPL mutation effect on JAK2 46/1 haplotype frequency in JAK2V617F-negative myeloproliferative neoplasms. Leukemia 24: 859-860.

Senyuk V, Rinaldi CR, Li D and Cattaneo F (2009). Consistent up-regulation of STAT3 independently of Jak2 mutations in a new murine model of essential thromcocythemia. Cancer Res. 69: 262-271.

Shuai K (2006). Regulation of cytokine signaling pathways by PIAS proteins. Cell Res. 16: 196-202.

Tefferi A (2006). Essential thrombocythemia: scientific advances and current practice. Curr. Opin. Hematol. 13: 93-98.

Teofili L, Martini M, Cenci T, Petrucci G, et al. (2007). Different STAT-3 and STAT-5 phosphorylation discriminates among Ph-negative chronic myeloproliferative diseases and is independent of the V617F JAK-2 mutation. Blood 110: 354-359.

Teofili L, Martini M, Cenci T, Guidi F, et al. (2008). Epigenetic alteration of SOCS family members is a possible pathogenetic mechanism in JAK2 wild type myeloproliferative diseases. Int. J. Cancer 123: 1586-1592.

Ueda M, Ota J, Yamashita Y, Choi YL, et al. (2003). DNA microarray analysis of stage progression mechanism in myelodysplastic syndrome. Br. J. Haematol. 123: 288-296.

Valentino L and Pierre J (2006). JAK/STAT signal transduction: regulators and implication in hematological malignancies. Biochem. Pharmacol. 71: 713-721.

Wernig G, Mercher T, Okabe R, Levine RL, et al. (2006). Expression of Jak2V617F causes a polycythemia vera-like disease with associated myelofibrosis in a murine bone marrow transplant model. Blood 107: 4274-4281.

Zhang Q, Raghunath PN, Xue L, Majewski M, et al. (2002). Multilevel dysregulation of STAT3 activation in anaplastic lymphoma kinase-positive T/null-cell lymphoma. J. Immunol. 168: 466-474. 